\title{
Contenido de sangre residual en el cordón umbilical postnacimiento en prematuros y niños de término
}

\author{
Post-Birth umbilical cord blood content in premature and term infants
}

Dora Salazar Herrera, ${ }^{*}, \neq$ Tonatiuh Fernando Ferreira Jaime, $\$$ Gilberto Guzmán Valdivia Gómez, II José Núñez del Prado Alcoreza, ${ }^{*, \neq}$ Pedro Orozco del Pino, " Alberto Orozco Gutiérrez ${ }^{*, \neq}$

\section{Resumen}

Objetivo: Determinar el contenido sanguíneo del cordón umbilical postnacimiento y su relación con el tamaño y el tiempo de corte mediante el análisis de las posibles implicaciones clínicas. Material y métodos: Se determinó la cantidad de sangre residual en el cordón umbilical de 252 neonatos en relación con la edad gestacional, la longitud del cordón y el tiempo de corte. Resultados: El contenido promedio de sangre en el cordón fue de $1.2 \mathrm{~mL} / \mathrm{kg} /$ peso o $0.19 \mathrm{~mL} / \mathrm{cm}$ de cordón. En los cordones mayores de $25 \mathrm{~cm}$ encontramos $2.29 \mathrm{~mL} / \mathrm{kg}$ y en los menores de $25 \mathrm{~cm} 0.79 \mathrm{~mL} / \mathrm{kg}$. Con corte tardío se obtuvo $2.27 \mathrm{~mL} / \mathrm{kg}$ y con corte temprano 2.34 $\mathrm{mL} / \mathrm{kg}$. En los prematuros la cantidad de sangre con cordón < a $25 \mathrm{~cm}$ fue de $0.89 \mathrm{~mL} / \mathrm{kg}$ y con cordón > $25 \mathrm{~cm}$ de 3.09 $\mathrm{mL} / \mathrm{kg}$, aunque sólo fueron cinco niños de este grupo. En los de término con cordón $<25 \mathrm{~cm}$ fue de $0.78 \mathrm{~mL} / \mathrm{kg}$ y $>$ a 25 $\mathrm{cm}$ de $2.22 \mathrm{~mL} / \mathrm{kg}$. Conclusiones: El cordón umbilical con longitud mayor de $25 \mathrm{~cm}$ puede proporcionar una transfusión de $2.29 \mathrm{~mL} / \mathrm{kg}$, el tiempo de corte tiene poco impacto en la cantidad total obtenida.

Palabras clave: Sangre de cordón, ordeñamiento del cordón umbilical, reanimación neonatal.

\section{INTRODUCCIÓN}

La reanimación neonatal está directamente relacionada con la morbimortalidad, ${ }^{1}$ uno disminuyó de 35\% a 8\%. ${ }^{1}$

El manual de reanimación neonatal de la AAP y AHA en su séptima edición recomienda el corte de cordón

\begin{abstract}
Objective: Determine the blood content of the post-birth umbilical cord and its relation to size and cut-off time as well as analyze the possible clinical implications. Material and methods: The amount of residual blood in the umbilical cord of 252 infants was used to estimate confidence intervals of the mean blood content of the post-birth umbilical cord in relation to gestational age, cord length and cut time. Results: The average blood content in the cord was $1.2 \mathrm{~mL} / \mathrm{kg} /$ weight or $0.19 \mathrm{~mL} / \mathrm{cm}$ in the 252 patients. In cords that are greater than $25 \mathrm{~cm}$ long we find $2.29 \mathrm{~mL} / \mathrm{kg}$ and in those less than $25 \mathrm{~cm}$ long we find $0.79 \mathrm{~mL} / \mathrm{kg}$. When using late clamping we obtained $2.27 \mathrm{~mL} / \mathrm{kg}$ and with an early cut $2.34 \mathrm{~mL}$ $\mathrm{kg}$. In premature infants, the amount of blood with cord length < $25 \mathrm{~cm}$ was $0.89 \mathrm{~mL} / \mathrm{kg}$ and with cord length $>25 \mathrm{~cm}$ of $3.09 \mathrm{~mL} /$ $\mathrm{kg}$ although there were only five children in this group. In the case of full-term births with cord length $<25 \mathrm{~cm}$, the amount of blood was $0.78 \mathrm{~mL} / \mathrm{kg}$ and $2.22 \mathrm{~mL} / \mathrm{kg}$ for the case of cord length $>25$ $\mathrm{cm}$. Conclusions: The umbilical cord with a length greater than $25 \mathrm{~cm}$ can provide a transfusion of $2.29 \mathrm{~mL} / \mathrm{kg}$, cord clamping time has little impact on the blood total amount obtained.
\end{abstract}

Keywords: Cord blood, umbilical cord milking, neonatal resuscitation.
* Departamento de Neonatología. Hospital Ángeles Pedregal, Ciudad de México.

₹ Curso de Subespecialización en Neonatología. Facultad Mexicana de Medicina. Universidad La Salle. Ciudad de México.

$\S$ Neonatólogo, Nuevo Sanatorio Durango, Ciudad de México.

॥ Jefe de Investigación, Facultad Mexicana de Medicina. Universidad La Salle. Ciudad de México.

" Departamento de Bioestadística. Universidad de Michigan, Ann Arbor, Estados Unidos.
Correspondencia:

Dr. Alberto Orozco Gutiérrez

Correo electrónico: orozcogutierrezalberto@gmail.com

Aceptado: 14-11-2019.

www.medigraphic.com/actamedica entre 30 y 40 segundos después del nacimiento así como la "ordeña" del cordón, ya que el volumen transfundido es necesario para la expansión adecuada del lecho vascular pulmonar. Aquí mismo se menciona que el tiempo ideal de corte del cordón no está estudiado en niños con asfixia y estado de choque y no deben retrasarse 
las maniobras habituales de reanimación por el corte de cordón. ${ }^{2}$

El tiempo de pinzamiento del cordón ha mostrado ser importante, ya que el lecho vascular pulmonar se dilata al nacer y requiere volumen sanguíneo para ello. ${ }^{2}$ Los trabajos de la Dra. Yao muestran que en los primeros 15 segundos después del nacimiento se transfunde aproximadamente $8 \mathrm{~mL} / \mathrm{kg}$ de peso. ${ }^{3,4}$

El "ordeñamiento" del cordón umbilical es un procedimiento en el cual el cordón se coloca entre dos dedos y la sangre es empujada hacia el recién nacido en un movimiento rápido y continuo, puede repetirse hasta lograr vaciar el cordón y proporcionar una transfusión de sangre propia; sin embargo, no se ha determinado qué cantidad de sangre se proporciona al niño con esta maniobra. ${ }^{2}$

El objetivo de este estudio es determinar el contenido sanguíneo del cordón y su relación con el tamaño y el tiempo de corte, verificar la utilidad potencial que puede tener la "ordeña" del cordón y analizar su potencial implicación clínica en la toma de decisiones en el tiempo de corte de cordón para la reanimación neonatal en niños con asfixia perinatal.

\section{MATERIAL Y MÉTODOS}

Se realizó un estudio descriptivo, observacional y transversal en el que se incluyeron neonatos sanos, mayores de 33 semanas de edad gestacional obtenidos por cualquier vía de nacimiento, que se agruparon en tres grupos; de 33 a 36 semanas de gestación (prematuros) y de más de 37 semanas de gestación (término) y población total.

Al momento del nacimiento el obstetra pinzó y cortó el cordón, el pediatra registró el tiempo de corte y después de los pasos iniciales de reanimación cortó el cordón umbilical a $2 \mathrm{~cm}$ de la base.
Posteriormente se procedió a "ordeñar" el cordón extirpado drenando la sangre en una jeringa graduada, y se midió la longitud del cordón con una cinta métrica.

La estadística descriptiva incluyó peso, semanas de gestación, tiempo de corte de cordón en segundos, contenido de sangre y longitud del cordón.

Se calcularon los intervalos de 95\% de confianza que se presentan en las Tablas 1 y 2.

\section{RESULTADOS}

Se estudiaron 252 neonatos, de los cuales 32 fueron prematuros y 220 de término. En la Tabla 1 se muestran las variables relacionadas con las semanas de gestación, peso al nacer, longitud del cordón umbilical, cantidad de sangre residual y su relación con el peso, longitud del cordón y tiempo de corte en los tres grupos.

En la muestra total (252 pacientes), la cantidad de sangre obtenida fue de $1.2 \mathrm{~mL} / \mathrm{kg} /$ peso o $0.19 \mathrm{~mL} / \mathrm{cm}$ de cordón. En los cordones mayores de $25 \mathrm{~cm}(\mathrm{n}=60)$ se extrajeron $2.29 \mathrm{~mL} / \mathrm{kg}$ (IC 95\% 1.96-2.62) de sangre residual y en los menores de $25 \mathrm{~cm}(\mathrm{n}=192) 0.79 \mathrm{~mL} / \mathrm{kg}$ (IC 95\% 0.71-0.88).

En el grupo de los prematuros, la cantidad promedio de sangre residual de cordón fue de $3.2 \mathrm{~mL} / \mathrm{kg}$ (IC 95\% 2.3-4.1); en los cordones mayores de $25 \mathrm{~cm}(n=5)$ fue de $3.09 \mathrm{~mL} / \mathrm{kg}$ (IC 95\% 2.19-3.98) y en los menores de 25 $\mathrm{cm}(\mathrm{n}=27)$ de $0.89 \mathrm{~mL} / \mathrm{kg}$ (IC 95\% 0.66-1.12).

En el grupo de los de término se extrajeron en promedio $3.5 \mathrm{~mL} / \mathrm{kg}$ (IC 95\% 3.0-3.9); con los cordones mayores de 25 $\mathrm{cm}(\mathrm{n}=55), 2.22 \mathrm{~mL} / \mathrm{kg}(\mathrm{IC} 95 \% 1.87-2.57)$ y en los menores de $25 \mathrm{~cm}(\mathrm{n}=165) 0.78 \mathrm{~mL} / \mathrm{kg}$ (IC 95\% 0.69-0.87); en ambos grupos, el corte temprano y tardío dieron cifras muy similares.

Como puede apreciarse, es importante la cantidad de sangre extraída del cordón proporcional a la longitud del mismo, factor importante para el manejo clínico (Tabla 2).

\begin{tabular}{|c|c|c|c|}
\hline Variable & $\begin{array}{l}\text { Muestra total }(\mathrm{N}=252) \\
\text { Media (IC 95\%) }\end{array}$ & $\begin{array}{l}\text { Prematuros }(n=32) \\
\text { Media }(\text { IC 95\%) }\end{array}$ & $\begin{array}{l}\text { De Término }(n=220) \\
\text { Media }(\text { IC } 95 \%)\end{array}$ \\
\hline Semanas de gestación & $37.9(37.8-38.1)$ & $35.8(35.1-36.1)$ & $38.3(38.1-38.4)$ \\
\hline Peso $(g)$ & $2,966(2,916.3-3,015.7)$ & $2,570.5(2,426.9-2,713.9)$ & $3,023.5(2,974.8-3,072)$ \\
\hline Longitud del cordón (cm) & $18(16.7-19.3)$ & $15.3(12.7-18.0)$ & $18.4(17.0-19.7)$ \\
\hline Contenido de sangre residual $(\mathrm{mL})$ & $3.4(3.1-3.8)$ & $3.2(2.3-4.1)$ & $3.5(3.0-3.9)$ \\
\hline $\begin{array}{l}\text { Sangre residual de acuerdo } \\
\text { con el peso }(\mathrm{mL} / \mathrm{kg})\end{array}$ & $1.2(1.0-1.3)$ & $1.2(0.87-1.6)$ & $1.14(1.0-1.27)$ \\
\hline $\begin{array}{l}\text { Sangre residual de acuerdo a la } \\
\text { longitud del cordón }(\mathrm{mL} / \mathrm{cm})\end{array}$ & $0.19(0.17-0.2)$ & $0.2(0.16-0.24)$ & $0.19(0.17-0.20)$ \\
\hline $\begin{array}{l}\text { Porcentaje de pacientes con tiempo } \\
\text { de corte de cordón }<15 \text { segundos }\end{array}$ & $23.1(18.5-29.1)$ & $21.9(7.5-36.2)$ & $24.1(18.4-29.7)$ \\
\hline
\end{tabular}


Tabla 2: Diferencias del contenido de sangre entre longitud de cordón $<25$ y > 25 cm. N = 252 .

\begin{tabular}{|c|c|c|c|c|}
\hline & \multicolumn{4}{|c|}{ Contenido de sangre en cordón (mL/kg) } \\
\hline & $\begin{array}{c}<25 \mathrm{~cm} \\
\text { Media (IC 95\%) }\end{array}$ & $\mathrm{n}$ & $\begin{array}{c}>25 \mathrm{~cm} \\
\text { Media (IC 95\%) }\end{array}$ & $\mathrm{n}$ \\
\hline Muestra total & $0.79(0.71-0.88)$ & 192 & $2.29(1.96-2.62)$ & 60 \\
\hline Corte temprano & $1.09(0.82-1.36)$ & 42 & $2.34(1.77-2.91)$ & 18 \\
\hline Corte tardío & $0.71(0.64-0.79)$ & 150 & $2.27(1.86-2.68)$ & 42 \\
\hline Pretérmino & $0.89(0.66-1.12)$ & 27 & $3.09(2.19-3.98)$ & 5 \\
\hline Corte temprano & $1.53(0.9-2.15)$ & 5 & $2.33(\mathrm{NA})$ & 2 \\
\hline Corte tardío & $0.74(0.53-0.95)$ & 22 & 3.59 (NA) & 3 \\
\hline Término & $0.78(0.69-0.87)$ & 165 & $2.22(1.87-2.57)$ & 55 \\
\hline Corte temprano & $1.04(0.74-1.33)$ & 37 & $2.34(1.72-2.97)$ & 16 \\
\hline Corte tardío & $0.70(0.62-0.78)$ & 128 & $2.17(1.75-2.59)$ & 39 \\
\hline
\end{tabular}

\section{DISCUSIÓN}

La transfusión de sangre residual de cordón es un concepto que adquiere validez día a día, múltiples estudios han demostrado sus beneficios.

Existen estudios que han medido la cantidad de sangre que permanece en la placenta y la que se transfunde al bebe de acuerdo al tiempo de corte ${ }^{3,4} y$ otros estudios han medido el contenido de sangre del cordón de acuerdo a la longitud; un estudio en prematuros muy pequeños (645 g) reveló que la transfusión obtenida al ordeñar cordones de $30 \mathrm{~cm}$ de largo puede llegar a ser de $17.7 \mathrm{~mL} / \mathrm{kg} /$ peso, $^{5}$ por esta razón decidimos en nuestro estudio determinar la cantidad de sangre encontrada relacionando la longitud de cordón y el peso, este concepto es claro si consideramos que la relación de $30 \mathrm{~cm}$ de cordón en un niño de 645 g sería el equivalente a la transfusión en un niño de $3 \mathrm{~kg}$ de la sangre de un cordón de $1.39 \mathrm{~m}$ de longitud.

Gupta en un estudio en prematuros demostró que el tiempo del pinzamiento del cordón es un factor relacionado con el riesgo de presentar anemia e hiperbilirrubinemia en las primeras horas de vida hasta los tres meses ${ }^{6}$ y el metaanálisis de Rabe reveló que el corte tardío disminuyó la anemia RR 0.61, IC 95\%, 0.46-0.81, la hemorragia intraventricular RR 0.59, IC 95\%, 0.41-0.85 y la enterocolitis necrosante (RR 0.62, IC 95\%, 0.43-0.90) demostrando un efecto benéfico en el corte tardío del cordón. ${ }^{7}$

Otro estudio en niños de término detectó que la expresión del cordón umbilical de más de $25 \mathrm{~cm}$ aumenta la presión arterial en las primeras 48 horas de vida y la hemoglobina a las seis semanas en $10 \%$, pero no determinó la cantidad transfundida; sin embargo, el concepto de que la presión arterial puede incrementarse con la expresión del cordón es muy prometedor. ${ }^{8}$
Otro estudio demostró que la posición del niño con respecto a la madre no influía en la transfusión o plazo como anemia, hiperbilirrubinemia, cambios en el flujo cerebral o policitemia. ${ }^{9}$

Al comparar el ordeñamiento con el retraso del corte de cordón se ha demostrado que existe paso de sangre y la diferencia en hematocrito y ferritina a las seis semanas no existe, este estudio proporcionó bases teóricas para recomendar el ordeñamiento del cordón cuando no existe posibilidad de esperar para el corte por la urgencia de la reanimación ${ }^{10}$ y no existió diferencia empleando el ordeñamiento simple en comparación con el ordeñamiento múltiple. ${ }^{11}$

El estudio ya referido de Hosono en prematuros extremos con un peso de $800 \mathrm{~g},{ }^{5}$ en el que se observó que el volumen de sangre residual es de 0.05 a $0.2 \mathrm{~mL} / \mathrm{cm}$ coincide con nuestros resultados (prematuros: 0.04-0.47 $\mathrm{mL} / \mathrm{cm}$; término: 0.013-0.73 mL/cm; muestra global: $0.01-0.73 \mathrm{~mL} / \mathrm{cm})$. En nuestros resultados la longitud mayor de $25 \mathrm{~cm}$ proporciona una transfusión en niños de término de $2.23 \mathrm{~mL} / \mathrm{kg}$, que sumada a los $8 \mathrm{~mL}$ que se transfunden con el corte a los 15 segundos, de acuerdo a los trabajos de Yao, ${ }^{3}$ puede proporcionar una transfusión de $10 \mathrm{~cm} / \mathrm{kg} /$ peso.

Un dato muy importante es que en todos los grupos la transfusión el cordón fue de aproximadamente $0.19 \mathrm{~mL} / \mathrm{cm}$.

En este estudio el tiempo de corte de cordón mostró poca importancia para fines de transfusión. En niños prematuros aparentemente el tiempo de corte es una variable que puede influir; sin embargo, sólo hubo seis prematuros con corte tardío de cordón, ésta es una limitante de nuestro estudio, ya que en este grupo nuestros resultados deben ser tomados con cautela y corroborados con un mayor número de casos. 
Existen autores que han mencionado el uso del corte tardío del cordón en niños asfixiados; sin embargo, la postura oficial de la Academia Americana de Pediatría es que el tiempo de corte de 30 segundos o más puede retrasar la reanimación, por lo que se ha sugerido que el "ordeñamiento" del cordón puede ser una alternativa útil, limitada hasta el momento por una falta de estandarización del procedimiento y de estudios para recomendar esta técnica como un procedimiento de rutina en niños asfixiados. ${ }^{3,8,12}$

En un trabajo experimental publicado en 2018 se demostró que las ovejas asfixiadas con corte temprano del cordón umbilical tuvieron mayor riesgo de daño cerebral manifestado por la reducción de las proteínas de barrera en la barrera hematoencefálica y un incremento de la pérdida de proteínas en la sustancia blanca y gris. ${ }^{13}$

La asfixia perinatal severa se acompaña de hipotensión arterial bajo gasto cardiaco y bradicardia e iniciar la reanimación en los primeros 30 segundos con expansión de volumen coincide con los criterios de la AHA, que indica que la reanimación debe iniciarse con medidas cardiovasculares.

La expansión de volumen es recomendada por la academia hasta el segundo minuto de la reanimación y se administran soluciones cristaloides por una vía instalada en lugar de lograr la expansión de volumen con la propia sangre placentaria y sin necesidad de instalar un catéter.

Desde el punto de vista teórico cuando el corte de cordón se realiza inmediatamente de acuerdo a los estudios de Yao, se priva al niño de la posibilidad de recibir $8 \mathrm{~mL} /$ $\mathrm{kg} /$ peso que se transfunden ellos los primeros 15 segundos y $2.29 \mathrm{~mL} / \mathrm{kg} /$ peso que se transfunden por el ordenamiento, esta carga rápida puede ser muy importante en niños asfixiados y este estudio puede proporcionar una base para realizar estudios clínicos.

Nuestro estudio es limitado, no tiene implicación clínica, no analiza la evolución de los pacientes, sólo se cuantifica el volumen y se hace un análisis absolutamente teórico.

En este estudio se demostró que la longitud del cordón al momento del pinzamiento es la variable más importante para la magnitud de la transfusión al momento de ordeñar el cordón en el recién nacido tanto prematuro como de término.

\section{CONCLUSIONES}

En la mayoría de los casos la longitud del cordón mayor de $25 \mathrm{~cm}$ proporciona una transfusión de $2.29 \mathrm{~mL} / \mathrm{kg} /$ peso.

Creemos que en niños asfixiados podría ser importante retardar el tiempo de cordón entre 15 y 30 segundos mientras el obstetra proporciona los pasos iniciales de la reanimación como aspiración y estimulación, lo que de acuerdo a Yao proporciona $8 \mathrm{~mL} / \mathrm{kg} /$ peso, dejando una longitud mayor de $25 \mathrm{~cm}$; y "ordeñando" el cordón, la transfusión obtenida de $10 \mathrm{~mL} / \mathrm{kg}$ puede ser de mucha importancia para revertir el estado de choque que acompaña a la asfixia. Esta posibilidad debe ser estudiada y evaluada.

Estos hallazgos proporcionan una base científica para reevaluar la recomendación de la Academia Americana de Pediatría con respecto a la técnica de ordeñamiento del cordón y pueden ser de utilidad para proporcionar bases teóricas y así iniciar estudios clínicos en niños asfixiados.

\section{REFERENCIAS}

1. Gutiérrez A, Velásquez R, Iriarte M. Analysis of clinical course in term patients with early and delayed umbilical cord clamping after birth. The Internet Journal of Pediatrics and Neonatology. 2009; 11 (2): 1-6.

2. Kattwinkel J, Ades A, Colby C, Eichenwald EC, Ernst KD, Escobedo M et al. Neonatal resuscitation textbook. 7a edición. American Academy of Pediatrics y American Heart Association, 2016; p. 6.

3. Yao A, Hirvensalo M, Lind J. Placental transfusion-rate and uterine contraction. Lancet. 1968; 1: 380-383.

4. Yao AC. Distribution of blood between infant and placenta after birth. Lancet. 1969; 2: 871-873.

5. Hosono S, Hine K, Nagano N, Taguchi Y, Yoshikawa K, Okada T et al. Residual blood volume in the umbilical cord of extremely premature infants. Pediatr Int. 2015; 57 (1): 68-71.

6. Gupta R, Ramji S. Effect of delayed cord clamping on iron stores in infants born to anemic mothers: randomized controlled trial. Indian Pediatr. 2002; 39 (2): 130-135.

7. Rabe H, Diaz-Rossello JL, Duley L, Dowswell T. Effect of timing of umbilical cord clamping and other strategies to influence placental transfusion at preterm birth on maternal and infant outcomes. Cochrane Database Syst Rev. 2012 15; 8: CD003248.

8. Upadhyay A, Gothwal S, Parihar R, Garg A, Gupta A, Chawla D, Gulati IK. Effect of umbilical cord milking in term and near term infants: randomized control trial. Am J Obstet Gynecol. 2013; 208: 120.e1-6

9. Jaiswal P, Upadhyay A, Gothwal S, Singh D, Dubey K, Garg A, Vishnubhatala S. Comparison of two types of intervention to enhance placental redistribution in term infants: randomized control trial. Eur J Pediatr. 2015; 174 (9): 1159-1167.

10. Hosono S, Mugishima H, Takahashi S, Takahashi S, Masaoka N, Yamamoto T et al. One-time umbilical cord milking after cord cutting has same effectiveness as multiple-time umbilical cord milking in infants born at $<29$ weeks of gestation: a retrospective study. J Perinatol. 2015; 35 (8): 590-594.

11. Vain NE, Satragno DS, Gorenstein AN, Gordillo JE, Berazategui JP, Guadalupe AM et al. Effect of gravity on volume of placental transfusion: a multicentre, randomised, non-inferiority trial. Lancet. 2014; 384 (9939): 235-240.

12. Duley L, Drife J, Soe A, Weeks A. Clamping of the umbilical cord and placental transfusion. RCOG Scientific Impact Paper No. 14. 2015.

13. Polglase GR, Blank DA, Barton SK, Miller SL, Stojanovska V, Kluckow $M$ et al. Physiologically based cord clamping stabilises cardiac output and reduces cerebrovascular injury in asphyxiated near-term lambs. Arch Dis Child Fetal Neonatal Ed. 2018; 103: F530-F538. 